\title{
Photo-induced cold vapor generation with low molecular weight alcohol, aldehyde, or carboxylic acid for atomic fluorescence spectrometric determination of mercury
}

\author{
Chunfang Han • Chengbin Zheng • Jun Wang • \\ Guanglei Cheng $\cdot$ Yi Lv $\cdot$ Xiandeng Hou
}

Received: 3 September 2006 /Revised: 5 November 2006 / Accepted: 8 November 2006 / Published online: 10 January 2007

(C) Springer-Verlag 2007

\begin{abstract}
With UV irradiation, $\mathrm{Hg}^{2+}$ in aqueous solution can be converted into $\mathrm{Hg}^{0}$ cold vapor by low molecular weight alcohols, aldehydes, or carboxylic acids, e.g., methanol, formaldehyde, acetaldehyde, glycol, 1,2-propanediol, glycerol, acetic acid, oxalic acid, or malonic acid. It was found that the presence of nano- $\mathrm{TiO}_{2}$ more or less improved the efficiency of the photo-induced chemical/cold vapor generation (photo-CVG) with most of the organic reductants. The nano- $\mathrm{TiO}_{2}$-enhanced photo-CVG systems can be coupled to various analytical atomic spectrometric techniques for the determination of ultratrace mercury. In this work, we evaluated the application of this method to the atomic fluorescence spectrometric (AFS) determination of mercury in cold vapor mode. Under the optimized experimental conditions, the instrumental limits of detection (based on three times the standard deviation of 11 measurements of a blank solution) were around 0.02

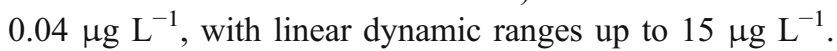
The interference of transition metals and the mechanism of the photo-CVG are briefly discussed. Real sample analysis using the photo-CVG-AFS method revealed that it was promising for water and geological analysis of ultralow levels of mercury.
\end{abstract}

Keywords Cold vapor generation · Mercury · Alcohol . Aldehyde $\cdot$ Carboxylic acid

C. Han $\cdot$ C. Zheng $\cdot$ J. Wang $\cdot$ Y. Lv $(\bowtie) \cdot$ X. Hou $(\bowtie)$

Ministry of Education Key Laboratory of Green Chemistry

and Technology, College of Chemistry, Sichuan University,

Chengdu, Sichuan 610064, China

e-mail: 1vy@scu.edu.cn

e-mail: houxd@scu.edu.cn

G. Cheng $\cdot$ X. Hou

Analytical and Testing Center, Sichuan University,

Chengdu, Sichuan 610064, China

\section{Introduction}

Mercury has become a widespread pollutant as a result of many industrial and agricultural applications [1]. The US EPA has currently set the upper limit of total mercury concentration in environmental water at $2 \mu \mathrm{g} \mathrm{L}^{-1}$ [2]. Consequently, method development for the cost-effective and sensitive determination of mercury in environmental and biological materials has been active in recent years [36]. Mercury cold vapor generation (CVG) has been most effectively used in the determination of mercury, and several reductants have been investigated for the reduction of $\mathrm{Hg}^{2+}$ to $\mathrm{Hg}^{0}$, e.g., stannous chloride [7], sodium cyanotrihydroborate(III) [8], and most frequently, sodium/ potassium tetrahydroborate (THB) [9]. In addition, electrolytic "reductant" could also be used for the generation of mercury cold vapor $[10,11]$.

In the new millennium, photo-CVG has been presented as an emerging research area. It is known that photogenerated electron donors, such as $\mathrm{H}$. and $\mathrm{CO}$, can contribute to the reduction of several metal ions to their corresponding vapor species. The photo-CVG of $\mathrm{SeH}_{2}$ from an aqueous slurry of $\mathrm{SeO}_{4}^{2-}$, formic acid, and $\mathrm{TiO}_{2}$ was firstly reported, without further analytical application, in 2000 [12]. Later in 2004, Wang et al. [13] studied the photo-induced pre-reduction of $\mathrm{Se}(\mathrm{VI})$ to $\mathrm{Se}(\mathrm{IV})$ by formic acid in the presence of nano- $\mathrm{TiO}_{2}$ as a catalyst, and THB was used in the generation of selenide. The same research group further demonstrated that photo-induced pre-reduction could also be applied to an electrochemical hydride generation system [14]. In fact, Sturgeon et al. [15, 16] has pioneered the research area of photo-CVG as an effective sample introduction method for analytical atomic spectrometry during the past two years. To date, selenium [17-19], nickel [20], arsenic [21], and mercury [22-25] have been 
converted to corresponding volatile species by using various photo-CVG systems. Sturgeon et al. [26] invented a combined spray chamber/UV photolysis unit to enhance sample introduction efficiency for several elements in the presence of $1-5 \%$ formic, acetic, and propionic acids, with enhancement factors of 2- to 40-fold for $\mathrm{Ag}$, As, Se, Sb, $\mathrm{Hg}, \mathrm{I}, \mathrm{Bi}, \mathrm{Pb}$, and $\mathrm{Sn}$ when the aerosol inside the chamber was illuminated with a $6-\mathrm{W}$ mercury pen lamp. Previous research in this group showed that the speciation analysis of inorganic mercury and methylmercury was possible by photo-CVG using formic acid only, with the use of the natural room light and alternatively UV radiation [22]. Using photo-CVG, we also demonstrated a green analytical method for the determination of mercury in wine or liquor samples, directly using the sample matrix as a reductant, without any additional chemical [23]. In our previous application studies, nano- $\mathrm{TiO}_{2}$ was not used. To this end, it was necessary to conduct a comprehensive study on the feasibility of using all low molecular weight alcohols, aldehydes, or carboxylic acids as the reductant for photo$\mathrm{CVG}$ for mercury, with and without nano- $\mathrm{TiO}_{2}$.

\section{Experimental}

\section{Instrumentation}

The instrumentation was detailed in our pervious work [22] and is therefore briefly summarized here. An AFS-2202 atomic fluorescence spectrometer (Beijing Haiguang Instrument Co., China) was used in this work for the determination of mercury. The default intermittent reactor was replaced by a laboratory-constructed flow-through photoreactor, as shown schematically in Fig. 1. Argon was used as a carrier/shield gas, and further atomization of mercury was unnecessary (cold vapor mode). The opti-

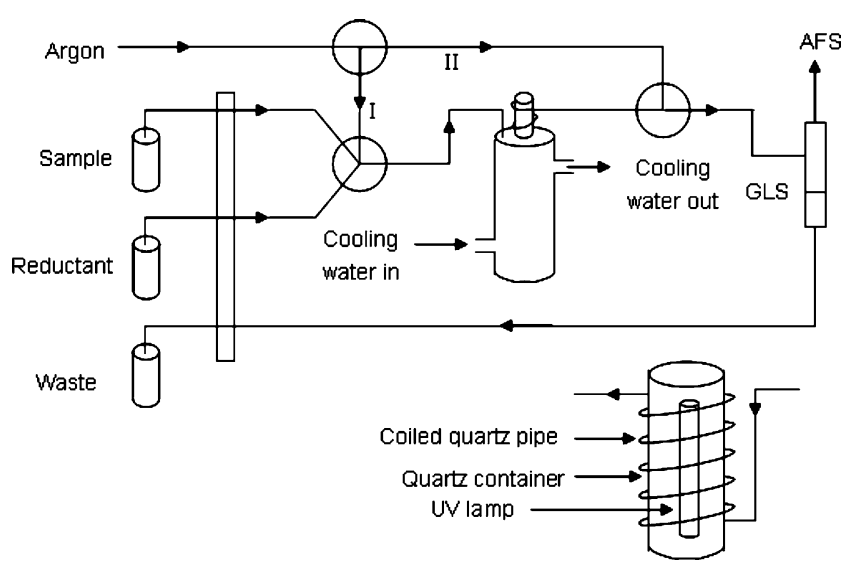

Fig. 1 Schematic diagram of the photo-CVG-AFS instrumentation. GLS gas/liquid separator. The lower drawing shows the photoreactor inside the water cooling unit mized instrumental parameters for the AFS determination of mercury are listed in Table 1.

Standard solutions and reagents

All reagents used were of at least analytical grade. Working solution of mercury was prepared daily by serial dilutions of a mercury standard stock solution of $1,000 \mathrm{mg} \mathrm{L}^{-1}$. Methanol, formaldehyde, acetaldehyde, acetic acid, glycol, 1,2-propanediol, glycerol, oxalic acid, or malonic acid (Xilong Chemical Co., Shantou, China) were tested as reductant. The blanks caused by these organic compounds are ignorable, much better than with THB systems. Commercial nano- $\mathrm{TiO}_{2}$ spherical particles $(10-30 \mathrm{~nm})$ were used as catalyst. Tap water samples were collected, after the tap was opened at full flow for $10 \mathrm{~min}$, from different sites on the Sichuan University campus. The certified reference water samples were purchased from the National Research Center for Certified Reference Materials (NRCCRM, Beijing, China), and the certified reference geological samples were purchased from Institute of Geophysical and Geochemical Exploration, Langfang, China.

Working procedure

The whole programmable operation procedure consisted of four steps. In step 1, mercury standard/sample solution and reductant were propelled into the photoreactor via the peristaltic pump for $20 \mathrm{~s}$ with a sampling volume of about $2.5 \mathrm{~mL}$. The argon flow passed through channel I to aid the sampling process. Nano- $\mathrm{TiO}_{2}$ was pre-mixed with the reductant and an ultrasonic bath was used to disperse the nano- $\mathrm{TiO}_{2}$ homogeneously before use. In step 2 , the pump was stopped, and the mixture of the mercury standard/ sample solution and the reductant in the photoreactor was irradiated with UV light for 5 to $20 \mathrm{~s}$, depending on the kind of reductant. At the same time, the argon flow was switched to channel II, and the sampling tubing was removed from the sample solution to the carrier solution (DDW). At the start of step 3, the argon flow was quickly

Table 1 Optimal instrumental parameters for the AFS determination of mercury

\begin{tabular}{ll}
\hline Parameter & Settings \\
\hline Mercury hollow cathode lamp & $253.7 \mathrm{~nm}$ \\
Lamp current & $30 \mathrm{~mA}$ \\
Voltage for photomultiplier tube & $-250 \mathrm{~V}$ \\
Observation height & $10 \mathrm{~mm}$ \\
Carrier argon flow rate & $500 \mathrm{~mL} \mathrm{~min}$ \\
Shield argon flow rate & $1,000 \mathrm{~mL} \mathrm{~min}$ \\
Quantification mode & Peak area \\
\hline
\end{tabular}


Table 2 Optimal concentrations of the reductant and nano- $\mathrm{TiO}_{2}$ and the irradiation time

\begin{tabular}{llll}
\hline Reductants & $\begin{array}{l}\text { Concentration } \\
(\mathrm{v} / \mathrm{v}, \%)^{\mathrm{a}}\end{array}$ & $\begin{array}{l}\mathrm{TiO}_{2} \\
\left(\mathrm{~g} \mathrm{~L}^{-1}\right)\end{array}$ & Irradiation time (s) \\
\hline Methanol & 10 & 2 & 20 \\
Formaldehyde & 20 & 3 & 10 \\
Acetaldehyde & 10 & 1 & 20 \\
Acetic acid & 10 & 3 & 20 \\
Glycol & 5 & 2 & 10 \\
1,2-Propanediol & 5 & 1 & 10 \\
Glycerol & 10 & 1 & 10 \\
Oxalic acid & 2 & 1 & 10 \\
Malonic acid & 5 & 1 & 5 \\
\hline
\end{tabular}

${ }^{\mathrm{a}} \mathrm{g} / 100 \mathrm{~mL}$ for oxalic acid and malonic acid

switched back to channel I, and the mixture was further propelled to the gas/liquid separator. After gas/liquid separation, the resultant gaseous mixture (mercury vapor and argon) was flushed into atomic fluorescence spectrometer for the measurement of atomic fluorescence of mercury. Step 4 was a 6-s delay to make the apparatus ready for the next measurement.

\section{Results and discussion}

\section{Optimization of photo-CVG conditions}

In our previous work $[22,23]$, we demonstrated that the reduction of $\mathrm{Hg}^{2+}$ could be achieved with formic acid or ethanol in the presence of natural or UV light, respectively. The conversion efficiency of the formic acid by the photoCVG system was not significantly improved by use of nano- $\mathrm{TiO}_{2}$; however, the ethanol system was. This led us to further investigate other similar organic reagents for use in photo-CVG for the determination of mercury, with and without nano- $-\mathrm{TiO}_{2}$ as a catalyst. Therefore, the reductants examined in the present work include methanol, formaldehyde, acetaldehyde, acetic acid, glycol, 1,2-propanediol, glycerol, oxalic acid, and malonic acid. Each of these can reduce $\mathrm{Hg}^{2+}$ to $\mathrm{Hg}^{0}$ to a certain extent with UV irradiation, and nano- $\mathrm{TiO}_{2}$ generally improves the conversion efficiency. The concentration of the reductant, the amount of $\mathrm{TiO}_{2}$, and the irradiation time were carefully optimized by using a $5 \mu \mathrm{g} \mathrm{L}^{-1} \mathrm{Hg}$ standard solution. The irradiation time was the time that the mixture of the sample and the reductant stayed in the photoreactor during step 2 when the UV lamp was on. We chose conditions giving the best signal-to-noise ratio and peak shape of the resultant atomic fluorescence signal for mercury. The optimized conditions for the photoCVG are summarized in Table 2.
Analytical figures of merit

Calibration curves were established with a series of $\mathrm{Hg}^{2+}$ standard solutions (at least five points in the range 2 $15 \mu \mathrm{g} \mathrm{L}^{-1}$ ) under the optimal experimental conditions. The calibration curves showed good linearity with linear correlation coefficients $\left(r^{2}\right)$ of at least 0.99 . The sensitivity was defined as the slope of the calibration curve obtained by least-squares regression analysis. The sensitivity enhancement factor was the ratio of the slope with nano- $\mathrm{TiO}_{2}$ to the slope without nano- $\mathrm{TiO}_{2}$. As shown in Table 3, the sensitivity enhancement factors are all larger than 1 , thus highlighting the effectiveness of the nano- $\mathrm{TiO}_{2}$ as a catalyst. However, the sensitivity (signal) enhancement is not large, and this means that, for the sake of simplicity, the nano- $\mathrm{TiO}_{2}$ may not be needed in cases where the sensitivity is already enough for the measurements required. The limits of detection (LODs, equivalent to $3 \sigma$ of 11 measurements of a blank solution) are also summarized in Table 3, with and without the nano- $\mathrm{TiO}_{2}$ catalyst. The LOD improvement factor was simply defined as [(LOD with $\left.\mathrm{TiO}_{2}\right) /(\mathrm{LOD}$ without $\left.\left.\mathrm{TiO}_{2}\right)\right] \times 100 \%$. As shown in Table 3, LOD is improved as a result of the enhancement of sensitivity when nano- $\mathrm{TiO}_{2}$ is added, except for the acetic acid system. With nano-TiO ${ }_{2}$, the LODs are basically the same as that exhibited by hydride generation AFS using THB $\left(0.03 \mu \mathrm{g} \mathrm{L}^{-1}\right)$ [5].

\section{Interference of transition metals}

One problem of the conventional hydride generation with THB is the interference from transition metal ions, mainly $\mathrm{Co}^{2+}, \mathrm{Cu}^{2+}$, and $\mathrm{Ni}^{2+}$. This may be due to the co-production of the metallic or colloidal state of these transition metal ions, and subsequently these metallic forms can catalytically decompose THB. In addition, those hydride-forming elements may also cause gas-phase interference in the

Table 3 Sensitivity enhancement and LODs for the determination of $\mathrm{Hg}$ using each of the proposed photo-CVG systems

\begin{tabular}{lcccc}
\hline Reductants & $\begin{array}{l}\text { Sensitivity } \\
\text { enhancement } \\
\text { factor }\end{array}$ & $\begin{array}{l}\mathrm{LOD} \text { with } \\
\mathrm{TiO}_{2} \\
\left(\mu \mathrm{g} \mathrm{L}^{-1}\right)\end{array}$ & $\begin{array}{l}\text { LOD } \\
\text { without } \\
\mathrm{TiO}_{2} \\
\left(\mu \mathrm{g} \mathrm{L}^{-1}\right)\end{array}$ & $\begin{array}{l}\text { LOD } \\
\text { improvement } \\
\text { factor }\end{array}$ \\
\hline Methanol & 1.4 & 0.04 & 0.05 & 1.3 \\
Formaldehyde & 1.3 & 0.03 & 0.04 & 1.3 \\
Acetaldehyde & 1.2 & 0.04 & 0.05 & 1.3 \\
Acetic acid & 1.4 & 0.03 & 0.02 & -0.6 \\
Glycol & 3.4 & 0.02 & 0.08 & 4.0 \\
1,2- & 3.4 & 0.03 & 0.1 & 3.3 \\
Propanediol & & & & 3.3 \\
Glycerol & 3.3 & 0.03 & 0.1 & 2.5 \\
Oxalic acid & 2.5 & 0.02 & 0.05 & 3.0 \\
Malonic acid & 3.1 & 0.02 & 0.06 & \\
\hline
\end{tabular}


Fig. 2 Left without nano- $-\mathrm{TiO}_{2}$ : a oxalic acid, b formaldehyde, c ethanol; right with nano- $\mathrm{TiO}_{2}$ in the organic reductant solution of $\mathbf{d}$ formaldehyde, $\mathbf{e}$ oxalic acid, f ethanol. The bottom overlapped traces are the cases when irradiating the organic species only prior to mixing with $\mathrm{Hg}^{2+}$; the upper traces (a-f) are the cases when irradiating the mixture

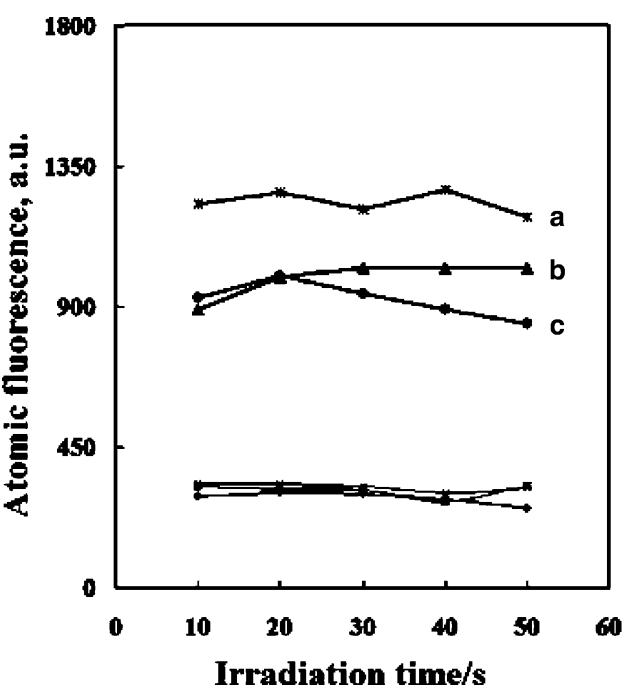

heated quartz tube. In the photochemical system, these interferences are eliminated or alleviated owing to the lower reducing ability of these alcohols, aldehydes, or carboxylic acids compared to THB, and the higher redox potential of mercury over the transition metal ions. In this work, the effects of $\mathrm{Cu}^{2+}, \mathrm{Co}^{2+}$, and $\mathrm{Ni}^{2+}$ on the generation and determination of $\mathrm{Hg}$ were investigated for each of the reductants. No significant interference from as high as $10 \mathrm{mg} \mathrm{L}^{-1} \mathrm{Cu}^{2+}, 50 \mathrm{mg} \mathrm{L}^{-1} \mathrm{Co}^{2+}$, and $100 \mathrm{mg} \mathrm{L}^{-1} \mathrm{Ni}^{2+}$ in a solution containing $5 \mu \mathrm{g} \mathrm{L}^{-1} \mathrm{Hg}$ was observed.

Mechanism for photo-CVG reaction

Many reports have discussed the possible mechanisms of photocatalytic reduction of mercury in the $\mathrm{UV} / \mathrm{TiO}_{2}$ system [27-31], and these are briefly summarized here. After absorption of sufficient energetic light $(\lambda<387 \mathrm{~nm})$ by $\mathrm{TiO}_{2}$, an electron is promoted to the higher energy conduction band $(\mathrm{cb})$, leaving a positively charged hole in the valence band $(\mathrm{vb})$. The photo-generated electrons $\left(\mathrm{e}_{\mathrm{cb}}^{-}\right)$ and holes $\left(\mathrm{h}_{\mathrm{vb}}^{+}\right)$migrate to surface of the semiconductor, where they are trapped by surface titanol groups, and $\mathrm{Hg}^{2+}$ is reduced to mercury atoms by the electrons, while the holes are scavenged by the carboxylic acid. The processes can be depicted by the following redox reactions:

$\mathrm{TiO}_{2}+h v \rightarrow \mathrm{e}_{\mathrm{cb}}^{-}+\mathrm{h}_{\mathrm{vb}}^{+}$
$\mathrm{Hg}^{2+}+2 \mathrm{e}^{-} \rightarrow \mathrm{Hg}$

$\mathrm{RCOOH}+\mathrm{h}^{+} \rightarrow \mathrm{CO}_{2}+\mathrm{H}_{2} \mathrm{O}+$ mineralacids

For an alcohol or an aldehyde, the following conversion to carboxylic acid may occur first:

$\mathrm{RCH}_{2} \mathrm{OH}+\mathrm{h}^{+} \rightarrow \mathrm{RCH}_{2} \mathrm{O} \cdot+\mathrm{H}^{+}$

$\mathrm{RCH}_{2} \mathrm{O} \cdot+\mathrm{RCH}_{2} \mathrm{OH} \rightarrow \mathrm{RCH}_{2} \mathrm{OH}+\mathrm{R} \cdot \mathrm{CHOH}$

$\mathrm{R} \cdot \mathrm{CHOH} \rightarrow \mathrm{RCHO}+\mathrm{H} \cdot$

$\mathrm{RCHO}+\cdot \mathrm{OH} \rightarrow \cdot \mathrm{RCHOHO}$

$\cdot \mathrm{RCHOHO}+\mathrm{O}_{2} \rightarrow \mathrm{RCOOH}+\cdot \mathrm{HO}_{2}$

The lifetime of free radicals is generally very short. The intensity of mercury atomic fluorescence, when only reductant is irradiated by UV light prior to mixing with $\mathrm{Hg}^{2+}$, should be far lower than that when a mixture of the reductant and $\mathrm{Hg}^{2+}$ is irradiated by UV light. To justify the assumption of short-lifetime radical mechanism, ethanol, formaldehyde, and oxalic acid were selected for experiments. The contents of organic species and nano- $\mathrm{TiO}_{2}$ were
Table 4 Analytical results and the recoveries of mercury from two tap water samples

${ }^{\text {a }}$ Sample number S-01

${ }^{\mathrm{b}}$ Sample number S-02

\begin{tabular}{lllll}
\hline Reductant & $\begin{array}{l}\text { Hg found, average } \pm 3 \mathrm{SD} \\
\left(n=3 ; \mu \mathrm{L} \mathrm{L}^{-1}\right)\end{array}$ & $\begin{array}{l}\text { Added } \\
\left(\mu \mathrm{g} \mathrm{L}^{-1}\right)\end{array}$ & $\begin{array}{l}\text { Total Hg found, average } \pm 3 \mathrm{SD} \\
\left(n=3 ; \mu \mathrm{L} \mathrm{L}^{-1}\right)\end{array}$ & Recovery (\%) \\
\hline Glycol & $1.60 \pm 0.03^{\mathrm{a}}$ & 1.00 & $2.75 \pm 0.06$ & 115 \\
1,2-Propanediol & $1.25 \pm 0.2^{\mathrm{b}}$ & 1.00 & $2.21 \pm 0.2$ & 96 \\
Glycerol & $1.01 \pm 0.2^{\mathrm{b}}$ & 1.00 & $1.99 \pm 0.2$ & 98 \\
Oxalic acid & $1.60 \pm 0.03^{\mathrm{a}}$ & 1.00 & $2.54 \pm 0.2$ & 115 \\
Malonic acid & $1.29 \pm 0.2^{\mathrm{b}}$ & 1.00 & $2.44 \pm 0.1$ & 94 \\
\hline
\end{tabular}


Table 5 Analytical results of mercury tap water samples in comparison with those by CV-AAS

vapor generation by using THB

\begin{tabular}{llcl}
\hline Sample & \multicolumn{2}{l}{ This method, average $\pm 3 \mathrm{SD}\left(n=3 ; \mu \mathrm{g} \mathrm{L}^{-1}\right)$} & $\begin{array}{l}\text { CV-AAS }{ }^{\mathrm{a}} \text { average } \pm 3 \mathrm{SD} \\
\left(n=3 ; \mu \mathrm{g} \mathrm{L}^{-1}\right)\end{array}$ \\
\cline { 2 - 3 } & Formaldehyde & Oxalic acid & \\
\hline S-03 & $0.49 \pm 0.03$ & $0.55 \pm 0.06$ & $0.57 \pm 0.03$ \\
S-04 & $0.48 \pm 0.06$ & $0.47 \pm 0.03$ & $0.57 \pm 0.06$ \\
S-05 & $0.68 \pm 0.03$ & $0.68 \pm 0.03$ & $0.80 \pm 0.06$ \\
S-06 & $0.84 \pm 0.03$ & $0.84 \pm 0.03$ & $0.97 \pm 0.06$ \\
S-07 & $0.88 \pm 0.03$ & $0.96 \pm 0.03$ & $1.04 \pm 0.06$ \\
\hline
\end{tabular}

their optimal concentrations, respectively, and the concentration of mercury was $5 \mu \mathrm{g} \mathrm{L}^{-1}$. The results are shown in Fig. 2. When only reductant is irradiated by UV light prior to mixing with $\mathrm{Hg}^{2+}$, the atomic fluorescence signal was minimal (the two overlapped traces in the bottoms of the two figures, respectively), and it is independent of the organic compound used. This experimental result supports the radical mechanism for one facet.

In the absence of nano- $-\mathrm{TiO}_{2}$, the organic acids may follow either of two different pathways during their photolytic decomposition, and produce hydrogen radicals, $\mathrm{H}_{2}$, and $\mathrm{CO}$ through reactions (9) and (10), taking formic acid as an example [32]. The produced hydrogen and/or carbon monoxide can reduce $\mathrm{Hg}^{2+}$ to mercury cold vapor. For alcohol and aldehyde, they may be converted to the relevant acids in the early stages of the photo-CVG process.

$\mathrm{HCOOH}+\mathrm{h} v \rightarrow \mathrm{H} \cdot+\cdot \mathrm{COOH} \rightarrow \mathrm{H}_{2}+\mathrm{CO}_{2}$

$\mathrm{HCOOH}+\mathrm{h} v \rightarrow \mathrm{HCO} \cdot+\cdot \mathrm{OH} \rightarrow \mathrm{CO}+\mathrm{H}_{2} \mathrm{O}$

\section{Analytical application}

To evaluate the applicability of the proposed photo-CVG systems in the analysis of real samples for mercury, two water samples were first analyzed for mercury by the proposed nano- $\mathrm{TiO}_{2}$-catalyzed photo-CVG-AFS with glycol, 1,2-propanediol, glycerol, oxalic acid, or malonic acid as a reductant. Recoveries of mercury of $94-115 \%$ were obtained, which is considered satisfactory for the low concentration level of mercury (Table 4). Furthermore, five water samples were analyzed using formaldehyde or oxalic acid as the reductant, and the analytical results were consistent with those obtained by cold vapor atomic absorption spectrometry (CV-AAS), considering the low level of mercury, as listed in Table 5.

Two certified reference water samples and three certified reference geological samples were also analyzed using formic acid and methanol, and the analytical results are listed in Table 6. The water sample of higher mercury concentration, i.e., GBW(E) 080393, was diluted prior to measurement. The geological samples were dissolved according to the literature procedure [33]. For the geological samples, the analytical results by using the proposed method with methanol were low (only around 60\% recovery) and are not listed in the table. The reason for the low recovery is unknown and awaits further investigation.

\section{Conclusions}

Several conclusions can be drawn from this work: (1) each of the nine low molecular weight alcohols, aldehydes, or carboxylic acids can be used as an effective reductant in photo-CVG for the determination of ultralow levels of mercury in aqueous solutions; (2) although the LODs do not outperform the traditional THB system for mercury, the photo-CVG systems suffer far less interferences from transition metal ions; and (3) this new analytical method is simple and cost-effective in terms of instrumentation or routine running.
Table 6 Analytical results of mercury in certified reference water or geological samples by the proposed method

${ }^{a} \mathrm{GBW}(\mathrm{E})$ is a series of water samples; the GSS series are soil samples with units of $\mu \mathrm{g} \mathrm{kg}^{-1}$

\begin{tabular}{llll}
\hline Sample $^{\mathrm{a}}$ & $\begin{array}{l}\text { Photo-CVG AFS, average } \pm \text { 3SD } \\
\left(n=3 ; \mu \mathrm{g} \mathrm{L}^{-1}\right)\end{array}$ & $\begin{array}{l}\text { Certified value, average } \pm \text { 3SD } \\
\left(n=3 ; \mu \mathrm{L} \mathrm{L}^{-1}\right)\end{array}$ \\
\cline { 2 - 3 } & Formic acid & Methanol & \\
\hline GBW(E) 080392 & $10.0 \pm 0.6$ & $10.2 \pm 0.3$ & $10 \pm 1.5$ \\
GBW(E) 080393 & $95.2 \pm 4.8$ & $97.3 \pm 7.3$ & $100 \pm 12$ \\
GSS3 & $61 \pm 2.0$ & - & $60 \pm 4.0$ \\
GSS4 & $523 \pm 60$ & - & $590 \pm 50$ \\
GSS5 & $258 \pm 42$ & - & $290 \pm 30$ \\
\hline
\end{tabular}


Acknowledgements We gratefully acknowledge the financial support of this work by the National Natural Science Foundation of China through Grant No.20375026 and Ministry of Education of China through Grant No.20030610068.

\section{References}

1. Harrington CF (2000) Trends Anal Chem 19:167-179

2. Environmental Protection Agency (2002) National primary drinking water regulations, chapt 1 . Code of federal regulations, title 40, vol 19. EPA, revised as of 1 July 2002, pp 428-429

3. Capelo JL, Maduro C, Mota AM (2004) J Anal At Spectrom 19:414-416

4. Tsalev DL (1999) J Anal At Spectrom 14:147-162

5. Long Z, Xin JJ, Hou XD (2004) Spectrosc Lett 37:263-274

6. Cava-Montesinos P, Ródenas-Torralba E, Morales-Rubio A, Cervera ML, de la Guardia M (2004) Anal Chim Acta 506:145-153

7. Gámiz-Gracia L, de Castro MDL (1999) J Anal At Spectrom 14:1615-1617

8. D’Ulivo A, Loreti V, Onor M, Pitzalis E, Zamboni R (2003) Anal Chem 75:2591-2600

9. Sturgeon RE, Mester Z (2002) Appl Spectrosc 56:202A-213A

10. Lin YH, Wang XR, Yuan DX, Yang PY, Huang BL (1992) J Anal At Spectrom 7:287-291

11. Denkhaus E, Golloch A, Guo XM, Huang BL (2001) J Anal At Spectrom 16:870-878

12. Kikuchi E, Sakamoto H (2000) J Electrochem Soc 147:4589-4593

13. Wang QQ, Liang J, Qiu JH, Huang BL (2004) J Anal At Spectrom 19:715-716

14. Liang J, Wang QQ, Huang BL (2005) Anal Bioanal Chem 381:366-372

15. Guo XM, Sturgeon RE, Mester Z, Gardner GJ (2003) Anal Chem 75:2092-2099
16. Guo XM, Sturgeon RE, Mester Z, Gardner GJ (2004) Anal Chem 76:2401-2405

17. Guo XM, Sturgeon RE, Mester Z, Gardner GJ (2003) Appl Organomet Chem 17:575-579

18. Guo XM, Sturgeon RE, Mester Z, Gardner GJ (2003) Environ Sci Technol 37:5645-5650

19. Figueroa R, García M, Lavilla I, Bendicho C (2005) Spectrochim Acta Part B 60:1556-1563

20. Guo XM, Sturgeon RE, Mester Z, Gardner GJ (2004) Appl Organomet Chem 18:205-211

21. Guo XM, Sturgeon RE, Mester Z, Gardner GJ (2005) J Anal At Spectrom 20:702-708

22. Zheng CB, Li Y, He YH, Ma Q, Hou XD (2005) J Anal At Spectrom 20:746-750

23. Li Y, Zheng CB, Ma Q, Wu L, Hu CW, Hou XD (2006) J Anal At Spectrom 21:82-85

24. Bendl RF, Madden JT, Regan AL, Fitzgerald N (2006) Talanta 68:1366-1370

25. Li HM, Zhang Y, Zheng CB, Wu L, Lv Y, Hou XD (2006) Anal Sci 22:1361-1365

26. Sturgeon RE, Willie SN, Mester Z (2006) J Anal At Spectrom 21:263-265

27. Skubal LR, Meshkov NK (2002) J Photochem Photobiol A: Chem 148:211-214

28. Chenthamarakshan CR, Yang H, Ming Y, Rajeshwar K (2000) J Eletroanal Chem 494:79-86

29. Chen DW, Ray AK (2001) Chem Eng Sci 56:1561-1670

30. Brezová V, Tarábek P, Dvoranová D, Staško A, Biskupiě S (2003) J Photochem Photobiol A: Chem 155:179-198

31. Wang KH, Jehng JM, Hsieh YH, Chang CY (2002) J Hazard Mat B 90:63-75

32. Khriachtchev L, Macôas E, Pettersson M, Räsänen M (2002) J Am Chem Soc 124:10994-10995

33. Xin JJ, He RL, Yang QJ, Hou XD (2006) Chem Res Appl (Ch) $18: 442-444$ 